\title{
The Structure of Price Gouging among United States' Hospitals and Physicians
}

\author{
William Riley ${ }^{\star}$, Natasha Coult and Kailey Love
}

School for the Science of Health Care Delivery, Arizona State University, Tempe, AZ 85281, USA

\begin{abstract}
Payment to hospitals and physicians for healthcare services in the United States are based on two primary components: 1) the charges rendered for the service delivered, and 2) the amount reimbursed by the payer. The structure for charges and reimbursement patterns are independent and unrelated to one another. As a consequence, a gap is created between the amount that hospitals and physicians charge for a service and the amount that is paid for that service [1].

To further add to the complexity, there is also a substantial gradient in the amount paid for a service depending upon the payer source [1]. For example, among the three primary payer sources in the United States (governmental, commercial health insurance payers, and direct payment from the patient), Medicaid and Medicare pay less than the cost of inpatient hospital services ( $87 \%$ and $89 \%$ respectively) while commercial health insurance companies pay approximately $130 \%$ of the cost of inpatient hospital services [2]. Patients pay personally for hospital and physician services when there is a deductible, co-pay, or if they have no government or commercial health insurance coverage [3]. In effect, the highest amount is often paid out of pocket by patients who are often the least able to afford it $[4,5]$.
\end{abstract}

This circumstance emanates from the common, yet poorly understood health care billing practices known as surprise medical billing (SMB) and out of network (OON) billing. SMB occurs when patients attend in-network hospitals but are treated by out-of-network physicians, [6] while OON billing arises when a patient receives care from a provider not included in the insurance plan network [7]. Patients subjected to SMB and OON charges typically pay the greatest amount for services and have little ability to negotiate payment [4] Critics refer to this situation as price gouging: the practice of setting prices for services at unfair levels and typically implying exploitative practices [8].

Price gouging has been described as extortion by health care organizations [9] and denounced because it can cause significant financial hardship for patients [10]. The Federal Reserve reported that $44 \%$ of Americans would not be able to cover an unexpected $\$ 400$ expense [11], while a study in the New England Journal of Medicine revealed that 1 in 5 of emergency department visits involved SMB with an average cost of $\$ 600$ [1]. Moreover, a 2016 report by the Kaiser Family Foundation found that approximately a quarter of the population had problems paying medical bills in the past year [12] and a study in the American Journal of Medicine found that almost half of bankruptcies in the United States are associated with medical billing [13].

Price gouging in health care differs from price gouging in other industries. In health care, prices are established using a comprehensive list with tens of thousands of potentially billable items. This list is known as a charge master. However, providers rarely carry out regular reviews of their charge master prices [14], resulting in a major disconnect between costs and charges. This gap occurs because hospital and physician charge master lists are usually set with unrealistically high prices. Government payers impose much lower payments and commercial payers negotiate reduced payments. The patient is unfortunately caught in the middle of a poorly designed

\section{Publication History:}

Received: June 20, 2018

Accepted: July 16, 2018

Published: July 18, 2018

\section{Keywords:}

Price Gouging, Health Care Payment, Surprise Medical Billing, Out of Network Billing, Payment Policy

payer system and usually does not know his or her rights or how to navigate through it, leaving patients without health insurance vulnerable to paying the highest amount for care. Charge master pricing patterns have evolved over several decades and most hospital prices today do not have a reasonable relationship to the cost of care [14]. Price gouging does not reflect any reasonable value proposition for health care organizations nor is it ethical to perpetuate.

It is an expeditious time for health care leaders to fix this problem. Strategies to proactively address price gouging include: 1 ) developing a coherent plan to meet the total financial requirements of the organization while avoiding an unfair burden upon those least able to afford health care; 2 ) ensuring that charge master prices are based on sensible cost recovery rates rather than hyper inflated fees that bear no relation to costs or third-party payment schedules; and 3) creating a value proposition that can be understood by all patients.

If provider organizations fail to proactively address price gouging, state legislatures appear willing to impose a solution on health care providers. Bills to regulate price gouging have been introduced in most states and enacted in six states [15]. Leaving this issue to be resolved by state legislatures is not ideal for providers or patients.

Price gouging is an unseemly tradition and should be carefully scrutinized by hospital and physician leaders. Shining a light on price gouging will provide deeper insight into what it represents, how it works, and why hospitals and physicians should discontinue the practice. SMB has been viewed for too long as a problem without a solution and continuing its practice will greatly jeopardize the reputation and integrity of providers by the communities that trust them to do the right thing.

\section{Competing Interests}

This commentary is based on research funded in part by a health insurance company

*Corresponding Author: Dr. William Riley, School for the Science of Health Care Delivery, Arizona State University, Tempe, AZ 85281, USA, Tel: 602-8034228; Email: william.j.riley@asu.edu

Citation: Riley W, Coult N, Love K (2018) The Structure of Price Gouging among United States' Hospitals and Physicians. Int J Community Fam Med 3: 141. https://doi.org/10.15344/2456-3498/2018/141

Copyright: () 2018 riley et al. This is an open-access article distributed under the terms of the Creative Commons Attribution License, which permits unrestricted use, distribution, and reproduction in any medium, provided the original author and source are credited. 
Citation: Riley W, Coult N, Love K (2018) The Structure of Price Gouging among United States' Hospitals and Physicians. Int J Community Fam Med 3: 141. https://doi.org/10.15344/2456-3498/2018/141

\section{References}

1. Sanger-Katz M, Abelson R (2016) Surprise! insurance paid the E.R. but not the doctor. The New York Times.

2. Ginsburg PB, Hughes M, Adler L, Burke S, Hoagland GW, et al. (2012) What is driving U.S. health care spending? America's unsustainable health care cost growth. Bipartisan Policy Center.

3. Robert Wood Johnson Foundation (2011) What are the biggest drivers of cost in U.S. health care?

4. Claxton G, Rae M, Long M (2016) Employer health benefits 2016 annual survey. The Henry J Kaiser Family Foundation.

5. Kacik A (2017) Rising hospital prices boost bottom lines but hurt uninsured. Modern Healthcare.

6. Pollitz K (2016) Surprise medical bills. The Henry J Kaiser Family Foundation.

7. Cooper Z, Morton FS (2016) Out-of-network emergency-physician bills - an unwelcome surprise. N Engl J Med 375: 1915.

8. Price gouging (2017) Legal Dictionary Web site.

9. Hacker JS (2017) Why an open market won't repair american health care. The New York Times.

10. Hamel L, Norton M, Pollitz K, Levitt L, Claxton G, et al. (2016) The burden of medical debt: Results from the kaiser family foundation/new york times medical bills survey. The Henry J Kaiser Family Foundation.

11. Federal Reserve Board Issues Report on the Economic Well-Being of U.S Households (2017) Board of Governors of the Federal Reserve System.

12. Lawsky B (2012) An unwelcome surprise: How new yorkers are getting stuck with unexpected medical bills from out-of-network providers. New York State Department of Financial Services.

13. Himmelstein DU, Thorne D, Warren E, Woolhandler S (2009) Medical bankruptcy in the united states: Results of a national study. Am J Med 122: 741-746.

14. Nation GA III (2005) Obscene contracts: The doctrine of unconscionability and hospital billing of the uninsured. The Kentucky Law Journal 94: 101.

15. Cousart C (2016) Answering the thousand-dollar debt question: An update on state legislative activity to address surprise balance billing. National Academy for State Health Policy. 